\title{
A New Fuzzy Logic Based Adaptation Mechanism for MRAS Sensorless Vector Control Induction Motor Drives
}

\author{
S.M. Gadoue, D. Giaouris and J.W. Finch \\ Power Electronics, Drives and Machines Group, School of EECE, Newcastle University, UK \\ Shady.Gadoue@ncl.ac.uk, Damian.Giaouris@ncl.ac.uk, J.W.Finch@ncl.ac.uk
}

Keywords: induction motor, sensorless control, fuzzy logic.

\begin{abstract}
This paper proposes a novel adaptation mechanism to replace the classical PI controller used in model reference adaptive speed estimation schemes which are based on rotor flux. The proposed adaptation mechanism is based on a fuzzy logic strategy. A detailed experimental comparison between the new and conventional schemes is carried out in both open and closed loop sensorless modes of operation when a vector control drive is working at very low speed. Superior performance has been obtained using the new fuzzy logic adaptation mechanism in both modes of operations.
\end{abstract}

\section{Introduction}

Among different rotor speed estimation techniques, Model Reference Adaptive Systems (MRAS) schemes are the most common strategies employed due to their relative simplicity and low computational effort [1]. Rotor flux MRAS, first proposed by Schauder [2], is the most popular MRAS strategy and a lot of effort has been focused on improving the performance of this scheme. Flux pure integration problems and stator resistance sensitivity are the main problems associated with this scheme.

Pure integration for flux represents a crucial difficulty which may cause dc drift and initial condition problems [3, 4]. LowPass Filters (LPF) with low cut-off frequency have been proposed to replace the pure integrator [5]. This introduces phase and gain errors and delays the estimated speed relative to the actual, which may affect the dynamic performance of the drive $[6,7]$ in addition to inaccurate speed estimation below the cut-off frequency [4]. To overcome this problem, Karanayil et al [6] introduces a programmable cascaded low pass filter (PCLPF) to replace the pure integration by small time constant cascaded filters to attenuate the dc offset decay time. In [8], another technique is used where the rotor flux is estimated by defining a modified integrator having the same frequency response as the pure integrator at steady state. The Neural Network (NN) has been presented as an adaptive filter used for signal integration to eliminate the offset in the flux integration for the voltage model flux observer [9]. A nonlinear feedback integrator for drift and dc offset compensation has been proposed in [10]. Further research has tried to entirely replace the voltage model with a full order stator and rotor flux observer which reduces the scheme's simplicity [11] .

Online adaptation of the stator resistance can improve the performance of the MRAS sensorless drive at low speed. In [12], a simultaneous estimation of rotor speed and stator resistance is presented based on a parallel MRAS observer where both the reference and adaptive models switch roles based on two adaptive mechanisms.

A linear $\mathrm{NN}$ is also proposed to represent the conventional adaptive current model where the estimated speed represents one of the neural network weights $[5,13]$. A back propagation or a least square algorithm is used to update these weights online. Another approach is discussed in [7] using a sliding mode flux observers in the adaptive model and deduces a speed estimation law based on sliding mode theory.

The majority of adaptation schemes described in the literature employ a simple fixed gain linear PI controller to generate the estimated motor speed. Not much attention has been devoted to the study of other types of adaptation mechanisms used to minimize the speed tuning signal to obtain the estimated speed.

This paper addresses this point by presenting an experimental approach to implement a new nonlinear adaptation mechanism based on Fuzzy Logic (FL) to replace the conventional PI controller used in the conventional rotor flux based-MRAS speed observer. A FL controller is proposed as a nonlinear optimizer to minimize the speed tuning signal used for rotor speed estimation. A detailed experimental comparison between the new and conventional schemes is carried out in both open and closed loop sensorless modes of operation when the drive is working at very low speed. Superior performance has been obtained using the new FL adaptation mechanism in both modes of operations.

\section{Rotor flux MRAS observer}

The classical rotor flux MRAS speed observer shown in Fig.1 consists mainly of a reference model, an adaptive model and an adaptation scheme which generates the estimated speed. The reference model, usually expressed by the voltage model, represents the stator equation. It generates the reference value of the rotor flux components in the stationary reference frame from the monitored stator voltage and current components. The reference rotor flux components obtained from the reference model are given by $[2,4]$ : 


$$
\begin{aligned}
& p \psi_{r d}=\frac{L_{r}}{L_{m}}\left\{v_{s d}-R_{s} i_{s d}-\sigma L_{s} p i_{s d}\right\} \\
& p \psi_{r q}=\frac{L_{r}}{L_{m}}\left\{v_{s q}-R_{s} i_{s q}-\sigma L_{s} p i_{s q}\right\}
\end{aligned}
$$

The adaptive model, usually represented by the current model, describes the rotor equation where the rotor flux components are expressed in terms of stator current components and the rotor speed. The rotor flux components obtained from the adaptive model are given by [2, 4]:

$$
\begin{aligned}
& p \hat{\psi}_{r d}=\frac{L_{m}}{T_{r}} i_{s d}-\frac{1}{T_{r}} \hat{\psi}_{r d}-\hat{\omega}_{r} \hat{\psi}_{r q} \\
& p \hat{\psi}_{r q}=\frac{L_{m}}{T_{r}} i_{s q}-\frac{1}{T_{r}} \hat{\psi}_{r q}+\hat{\omega}_{r} \hat{\psi}_{r d}
\end{aligned}
$$

Finally, the adaptation scheme generates the value of the estimated speed to be used in such a way as to minimize the error between the reference and estimated fluxes. In the classical RF-MRAS scheme, this is performed by defining a speed tuning signal, $\varepsilon_{\omega}$, to be minimized by a PI controller which generates the estimated speed which is fed back to the adaptive model. The expressions for the speed tuning signal and the estimated speed can be given as [4]:

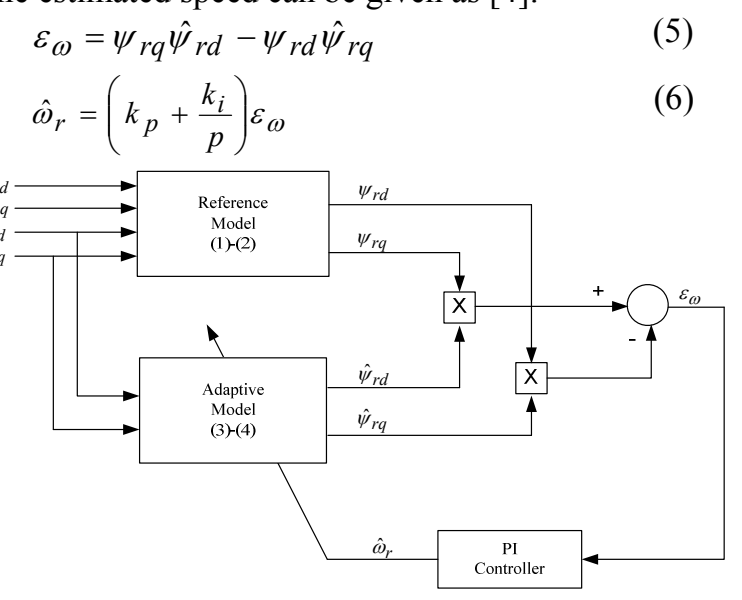

Fig.1 Conventional MRAS speed observer

\section{Fuzzy Logic MRAS observer}

Fuzzy logic control (FLC) has been found to be excellent in dealing with systems that are imprecise, nonlinear, or timevarying and with uncertain or unknown parameter and structure variation. FLC is relatively easy to implement, indeed it does not need any mathematical model of the controlled system, making it popular for control, estimation and optimization problems. This is achieved by converting the linguistic control strategy of human experience and knowledge into an automatic control strategy [14].

For MRAS speed observer, the mechanism of the estimation of the rotor speed can be regarded as an optimization problem where the PI controller is generating a quantity, the estimated speed, in such a way as to minimize a specified error, which is the speed tuning signal in (5), in a feedback loop. Therefore, FLC can replace the conventional PI controller to solve the optimization problem.
The proposed FLC is a Mamdani-type rule base where the inputs are the speed tuning signal in (5) and its rate of change. These two inputs are multiplied by two scaling factors $K_{e}$ and $K_{d}$ respectively. The output of the controller is multiplied by a third scaling factor $K_{u}$ to generate the actual value of the rate of change of the estimated speed. Finally, a discrete integration is performed to get the value of the estimated speed. The choice of the values of the scaling factors greatly affects the performance of the FLC. Trial and error technique is used to tune these gains to ensure optimal performance of the controller [15]. Each variable of the FLC has seven membership functions. The following fuzzy sets are used: $\mathrm{NB}=$ NEGATIVE BIG, NM= NEGATIVE MEDUIM, NS= NEGATIVE SMALL, ZE = ZERO, PS= POSITIVE SMALL, $\mathrm{PM}=$ POSITIVE MEDUIM, $\mathrm{PB}=$ POSITIVE BIG. The membership functions of the FLC are shown in Fig. 2. Table 1 shows the fuzzy rule base with 49 rules [15]. The overall MRAS speed observer with FL speed estimation mechanism is shown in Fig. 3.

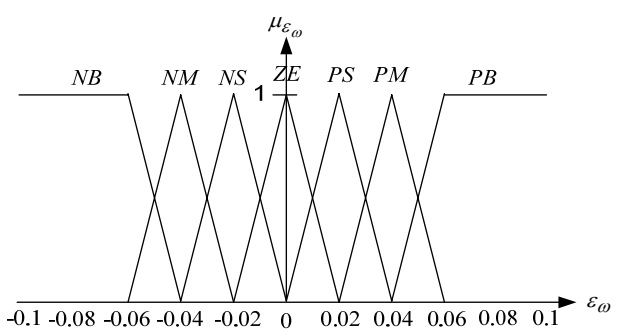

(a)

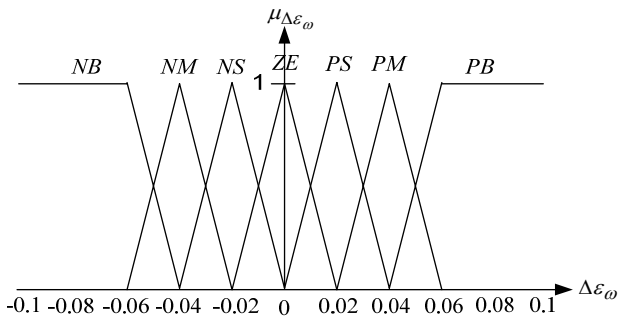

(b)

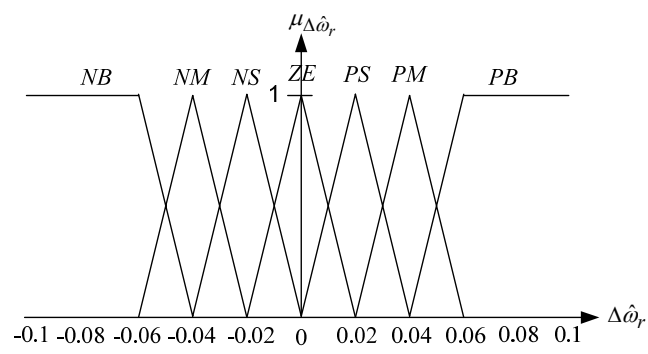

(c)

Fig. 2 Fuzzy controller input and output membership functions (a) error (b) error change (c) change in the estimated speed

\section{The experimental system}

The experimental platform consists of a $7.5 \mathrm{~kW}, 415 \mathrm{~V}$, delta connected three phase induction machine loaded by a $9 \mathrm{~kW}$, $240 \mathrm{~V}, 37.5$ A separately excited DC load machine to allow separate control of torque and speed. A $15 \mathrm{~kW}$ four quadrant DC drive from the Control Techniques "Mentor" range is 
used to control the DC machine to provide different levels of loading on the induction machine up to full load. The induction machine parameters are given in the Appendix.

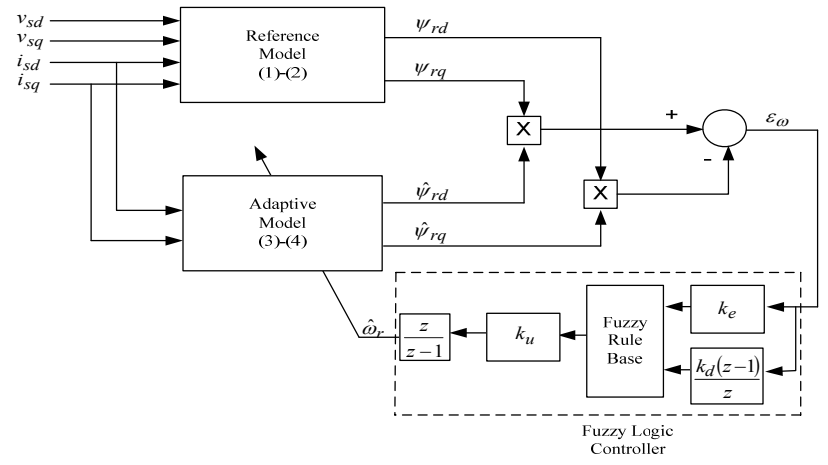

Fig.3 FL-MRAS speed observer

The AC drive power electronics consists of a 50A 3 Phase Diode Bridge and $1200 \mathrm{~V}$, 50A half bridge IGBT power modules. To control the induction motor a dSPACE system is used which contains a PowerPC 604e running at $400 \mathrm{MHz}$, and a Slave TMS320F240 DSP.

The actual motor speed is measured by a 5000 pulses/revolution speed encoder. The inverter switching frequency is $15 \mathrm{kHz}$ and the vector control is executed with the same sampling frequency. The observer and the speed control loop have a sampling frequency of $5 \mathrm{kHz}$ and the speed measurement is executed with a sampling frequency of $250 \mathrm{~Hz}$.

\begin{tabular}{|l|l|l|l|l|l|l|l|}
\hline$\varepsilon_{\omega}$ & NB & NM & NS & ZE & PS & PM & PB \\
\hline NB & NB & NM & NM & NS & NS & NS & ZE \\
\hline NM & NM & NM & NS & NS & NS & ZE & PS \\
\hline NS & NM & NM & NS & NS & ZE & PS & PM \\
\hline ZE & NB & NM & NS & ZE & PS & PM & PM \\
\hline PS & NS & NS & ZE & PS & PS & PM & PM \\
\hline PM & NS & ZE & PS & PS & PS & PM & PM \\
\hline PB & ZE & PS & PS & PM & PM & PB & PB \\
\hline \multicolumn{7}{|c}{ Table 1: Fuzzy controller rules } \\
\hline
\end{tabular}

During practical implementation of the MRAS scheme it was found necessary to cascade a low cut-off frequency HPF at the outputs of the voltage model (VM) to remove integrator drift and any initial condition problems. The cut-off frequency should be selected as low as possible since the purpose is just to remove the DC component and therefore a value of $1 \mathrm{~Hz}$ is chosen.

A simple dead time compensator similar to $[16,17]$ is implemented and reference voltages which are available in the control unit are used as the real stator voltages and will be used for voltage model flux observer in (1) and (2).

To use the FLC in real time with dSPACE card and Simulink, a two dimensional look-up table is generated from the FL toolbox in Matlab with a step size of 0.0005 for the inputs. The FLC implementation using a look-up table is shown in Fig.4 where the saturation limits for the input saturation blocks are set to 0.1 and -0.1 .

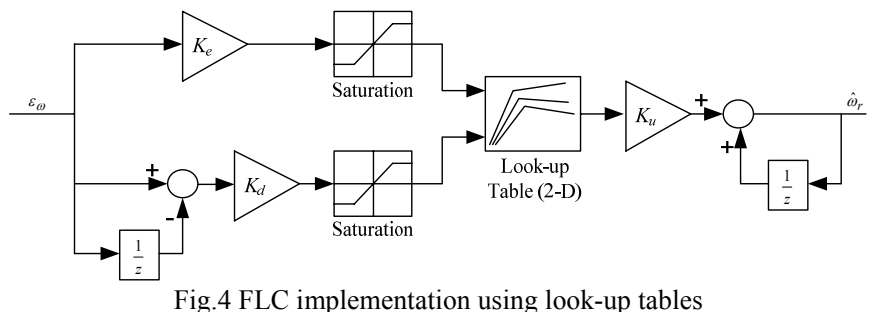

\section{Experimental Results}

Extensive experimental tests were carried out to compare between the two adaptation schemes, PI and FL, using an indirect vector control induction motor drive. The tests were performed in both open loop and sensorless modes of operation. Selected experimental results from these tests are shown in the following sections.

\subsection{Open loop performance}

The two adaptation schemes were tested in open loop when the drive is operated as encodered vector control, i.e. the encoder speed is used for speed control and rotor flux angle estimation. The drive was subjected to different reference speed changes at various load torque levels. To allow fair comparison the two controllers gains were tuned in such a way as to obtain similar steady state performance. The parameters of the PI controller are $K_{p}=10$ and $K_{i}=100$ while as these of the FLC are $k_{e}=0.01, k_{d}=1$ and $k_{u}=5$.

At low speed a steady state error in the estimated speed is observed for the MRAS observer. This is mainly due to the stator resistance mismatch between the motor and the observer. Moreover, since dead time effects cannot be completely removed even by complicated compensation schemes [3], the reference voltages used for the voltage model did not match the actual stator voltages across the machine terminals which represents another source for the steady state error in the estimated speed.

Figs. 5-6 show the speed estimation performance of both schemes for reference speed change from $100 \mathrm{rpm}$ to $50 \mathrm{rpm}$ at no load and for $25 \%$ load torque disturbance rejection at 60 rpm respectively. The FL scheme shows a better transient response compared to PI scheme which is due to an optimal speed tuning signal during transients.

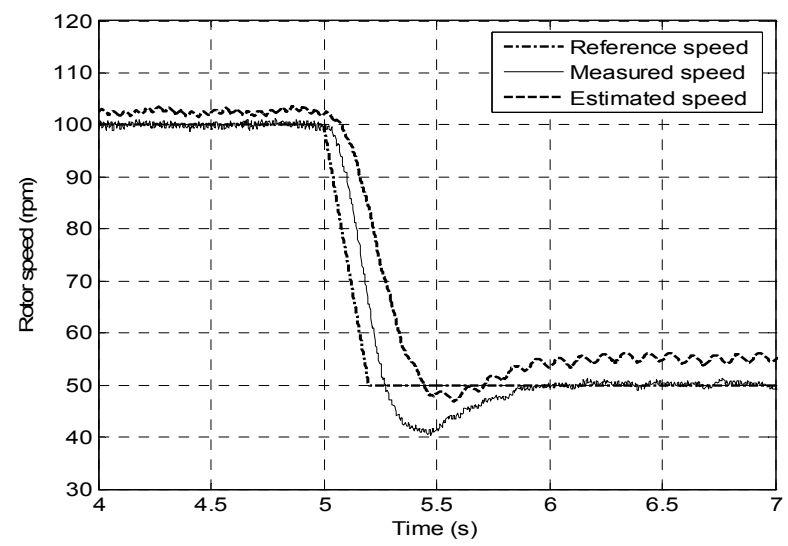

(a) 


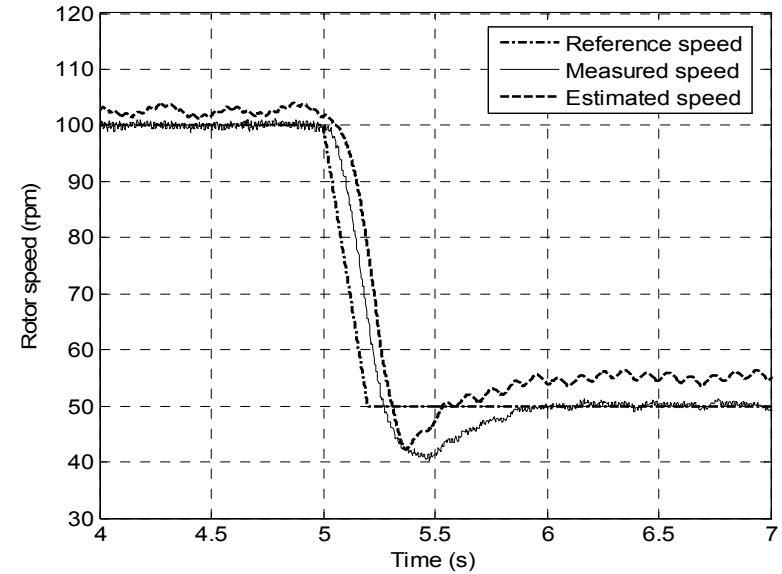

(b)

Fig. 5 Speed estimation performance at no load (a) MRAS PI (b) MRAS FL

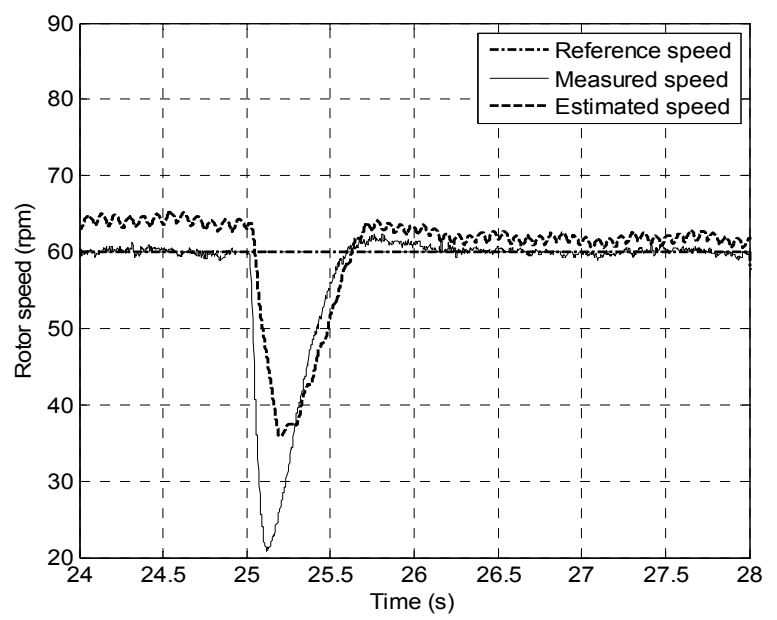

(a)

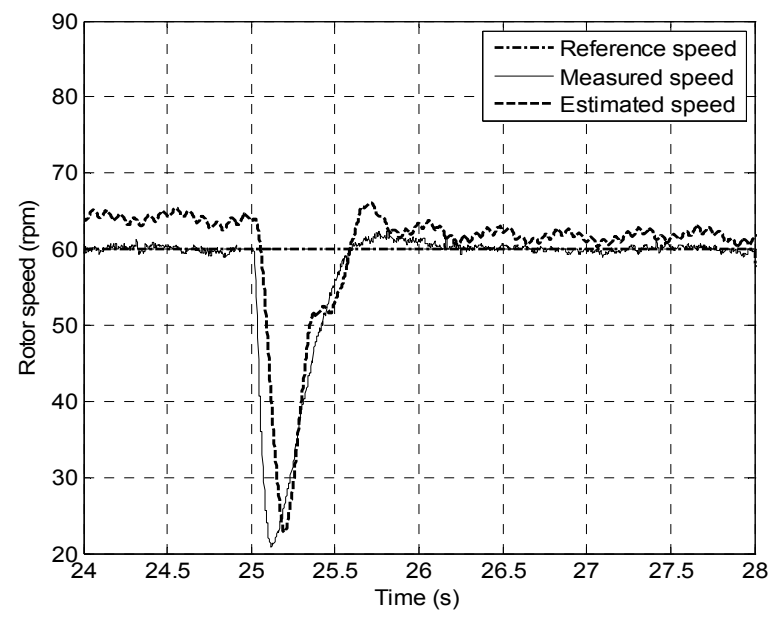

(b)

Fig. 6 Speed estimation performance for $25 \%$ load torque disturbance rejection (a) MRAS PI (b) MRAS FL

\subsection{Sensorless performance}

In these tests, the vector control drive is working in the closed loop sensorless mode where the estimated speed is used for both speed control and rotor flux orientation. The two schemes are compared when the drive is running at different operating conditions at very low speed.

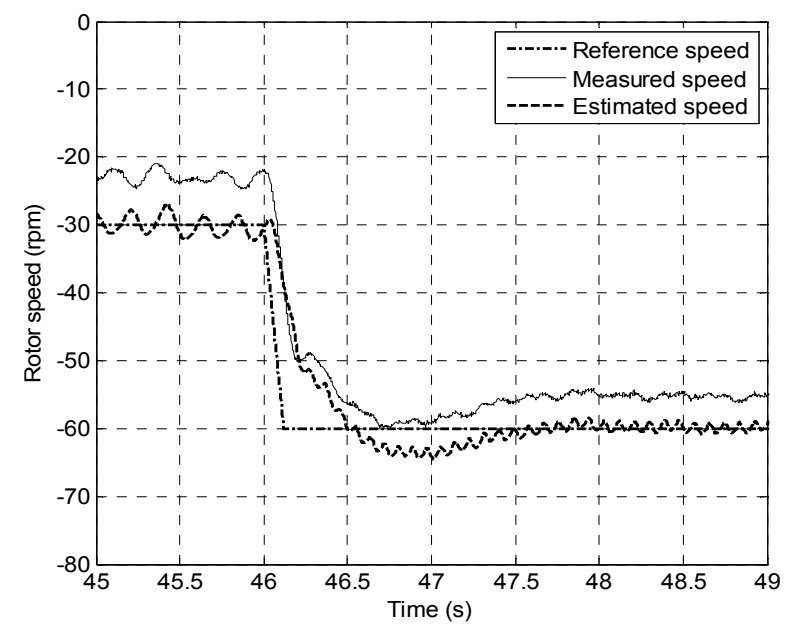

(a)

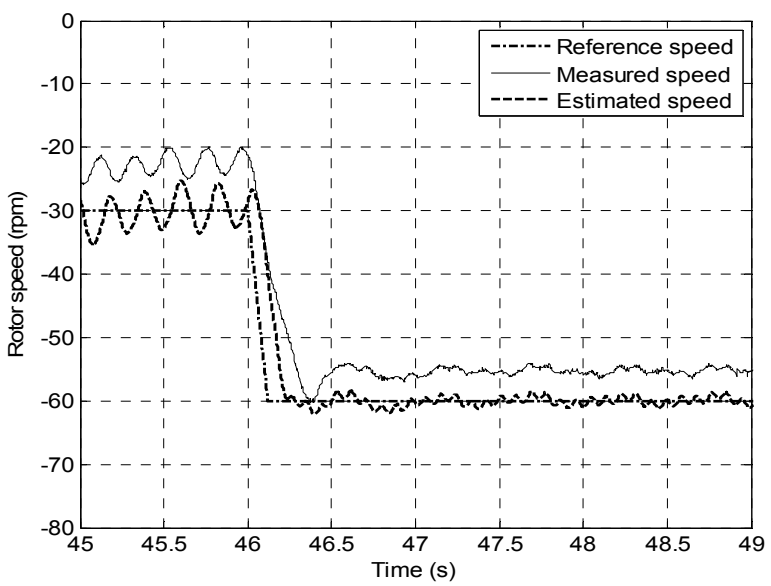

(b)

Fig. 7 Sensorless performance at no load (a) MRAS PI (b) MRAS FL Sensorless performance of both schemes is shown in Figs. 7-8 where the drive is subjected to reference speed change from $30 \mathrm{rpm}$ to $-60 \mathrm{rpm}$ at no load and a $25 \%$ load torque application at $100 \mathrm{rpm}$. Compared to the PI scheme, FL still shows a faster response during transients.

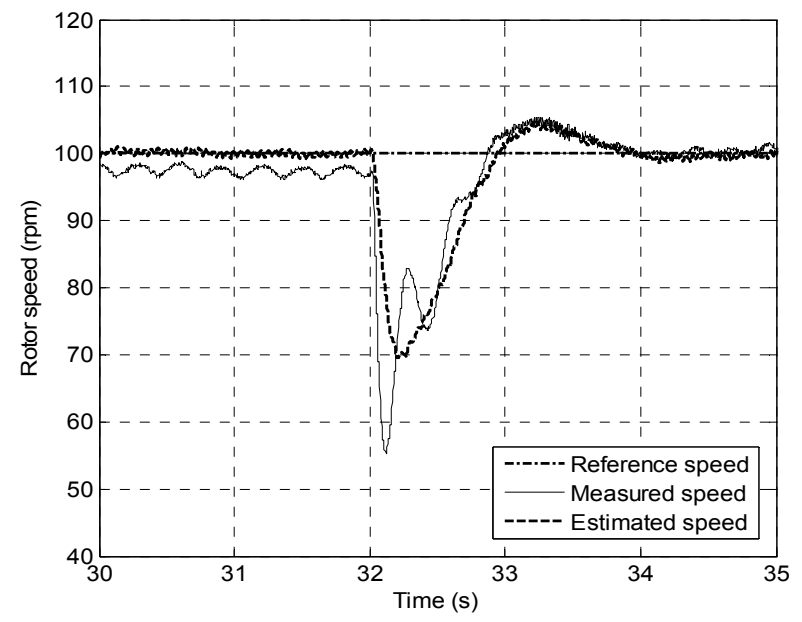

(a) 


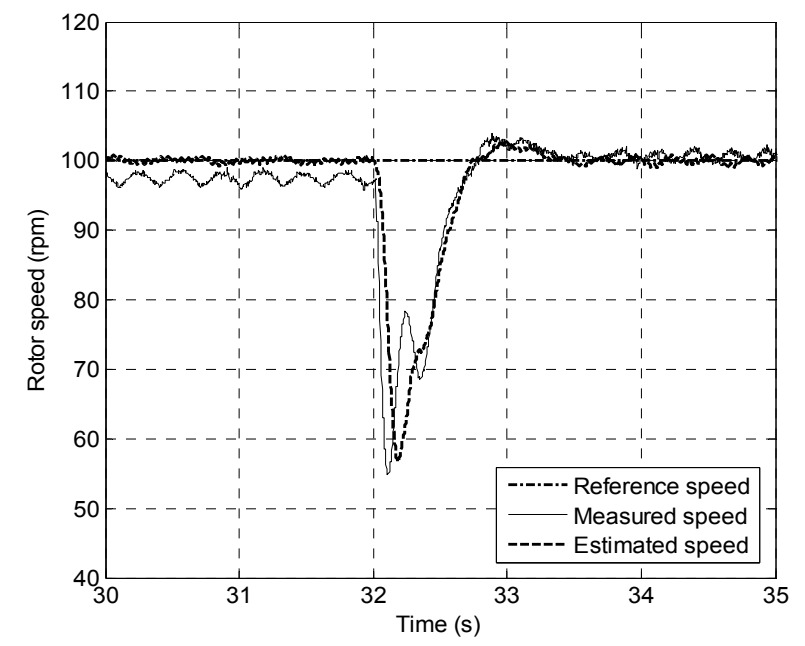

(b)

Fig. 8 Sensorless performance for load disturbance rejection (a) MRAS PI (b) MRAS FL

\section{Conclusion}

This paper addresses the problems of speed estimation at very low speed using a rotor flux-based MRAS speed observer and proposes a novel FL adaptation mechanism to replace the classical fixed gain PI controller. Parameter tuning of both schemes has been performed in such a way as to obtain similar steady state performance. A detailed comparison between the two schemes has been carried out using an indirect vector control induction motor drive. Application of Fuzzy Logic shows better transient performance as well as better load torque disturbance rejection in both open loop and closed loop sensorless modes of operation.

\section{Appendix}

Motor Parameters

$7.5 \mathrm{~kW}, 3$-phase, $415 \mathrm{~V}$, delta connected, $50 \mathrm{~Hz}, 4$ pole, Star equivalent parameters: $R_{s}=0.7767 \Omega, R_{r}=0.703 \Omega, L_{s}=$ $0.10773 \mathrm{H}, L_{r}=0.10773 \mathrm{H}, L_{m}=0.10322 \mathrm{H}, J=0.22 \mathrm{~kg} \cdot \mathrm{m}^{2}$

\section{References}

[1] M. Rashed and A. F. Stronach, "A stable back-EMF MRAS-based sensorless low speed induction motor drive insensitive to stator resistance variation," IEE Proceedings Electric Power Applications, vol. 151, pp. 685-693, 2004.

[2] C. Schauder, "Adaptive speed identification for vector control of induction motors without rotational transducers," IEEE Transactions on Industry Applications, vol. 28, pp. 1054-1061, 1992.

[3] J. Holtz and J. Quan, "Drift and parameter compensated flux estimator for persistent zero stator frequency operation of sensorless controlled induction motors," IEEE Transactions on Industry Applications, vol. 39, pp. 1052-1060, 2003.
[4] P. Vas, Sensorless Vector and Direct torque control. New York: Oxford University Press, 1998.

[5] L. Ben-Brahim, S. Tadakuma, and A. Akdag, "Speed control of induction motor without rotational transducers," IEEE Transactions on Industry Applications, vol. 35, pp. 844-850, 1999.

[6] B. Karanayil, M. F. Rahman, and C. Grantham, "An implementation of a programmable cascaded low-pass filter for a rotor flux synthesizer for an induction motor drive," IEEE Transactions on Power Electronics, vol. 19, pp. 257-263, 2004.

[7] M. Comanescu and L. Xu, "Sliding mode MRAS speed estimators for sensorless vector control of induction machine," IEEE Transactions on Industrial Electronics, vol. 53, pp. 146-153, 2006.

[8] M. Hinkkanen and J. Luomi, "Modified integrator for voltage model flux estimation of induction motors," IEEE Transactions on Industrial Electronics, vol. 50, pp. 818-820, 2003.

[9] M. Cirrincione, M. Pucci, G. Cirrincione, and G. Capolino, "A new adaptive integration methodology for estimating flux in induction machine drives," IEEE Transactions on Power Electronics, vol. 19, pp. 25-34, 2004.

[10] Q. Gao, C. S. Staines, G. M. Asher, and M. Sumner, "Sensorless speed operation of cage induction motor using zero drift feedback integration with MRAS observer," in Proc. European Conference on Power Electronics and Applications (EPE 2005), 2005.

[11] C. C. Lascu, I. Boldea, and F. Blaabjerg, "A modified direct torque control for induction motor sensorless drive," IEEE Transactions on Industry Applications, vol. 36, pp. 122-130, 2000.

[12] V. Vasic and S. Vukosavic, "Robust MRAS-Based algorithm for stator resistance and rotor speed identification," IEEE Power Engineering Review, vol. 21, pp. 39-41, 2001.

[13] M. Cirrincione and M. Pucci, "An MRAS-based sensorless high-performance induction motor drive with a predictive adaptive model," IEEE Transactions on Industrial Electronics, vol. 52, pp. 532- 551, 2005.

[14] P. Vas, Artificial-Intelligence-Based Electrical Machines and Drives-Application of Fuzzy, Neural, Fuzzy-Neural and Genetic Algorithm Based Techniques. New York: Oxford University Press, 1999.

[15] Y. Miloud and A. Draou, "Fuzzy logic based rotor resistance estimator of an indirect vector controlled induction motor drive," in Proc. IEEE 28th Annual Conference of the Industrial Electronics Society IECON 02, 2002.

[16] S. H. Kim, T. S. Park, J. Y. Yoo, G. T. Park, and N. J. Kim, "Dead time compensation in a vector-controlled induction machine," in Proc. Power Electronics Specialists Conference (PESC 98), 1998.

[17] L. Ben-Brahim, "On the compensation of Dead Time and Zero-Current crossing for a PWM-Invertercontrolled AC servo drive," IEEE Transactions on Industrial Electronics, vol. 51, pp. 1113-1117, 2004. 